\title{
Investigating the Distribution of Knowledge Structure in Extended Concept Mapping
}

\author{
Didik Dwi Prasetya ${ }^{1,2, *}$, Aryo Pinandito ${ }^{1,3}$, Yusuke Hayashi ${ }^{1}$, and Tsukasa \\ Hirashima $^{1}$
}

\begin{abstract}
${ }^{1}$ Department of Information Engineering, Graduate School of Engineering, Hiroshima University, Japan. ${ }^{2}$ Department of Electrical Engineering, Faculty of Engineering, Universitas Negeri Malang, Indonesia.

${ }^{3}$ Department of Information System, Faculty of Computer Science, Universitas Brawijaya, Indonesia.

${ }^{*}$ Corresponding author.Email: didikdwi@um.ac.id
\end{abstract}

\begin{abstract}
Extended concept mapping is a technique to integrate prior existing knowledge structure a new relevant information. Some researchers argue that the extended concept mapping approach is efficient in building a knowledge base and facilitating improved meaningful learning. However, no information has been presented regarding the distribution of knowledge structure. This study aimed to compare two extended concept mapping approaches and investigate the distribution of quality of students' knowledge structures. A total of 55 college students participated and divided into two groups, control and experimental. The students in the control group used the Extended Scratch-Build (ESB) technique, and those in the experimental group used the Extended Kit-Build (EKB) map. The results suggested that the students in the experimental group not only outperformed the control group in terms of quality of knowledge structure scores but also had an equitable distribution of achievement in material subtopics. Students who used the EKB were able to maintain performances consistently from the beginning to the end of material subtopics.
\end{abstract}

Keywords: Knowledge structure, extended concept mapping, Extended Kit-Build, Extended Scratch-Build

\section{INTRODUCTION}

Individual knowledge represents the collection of facts in the real world known by the individual. Cognitive psychologists suggest that the "essence of knowledge is structure" [1]. Knowledge requires a highly integrated conceptual structure among concepts to be knowledgeable and meaningful in the context domain. Knowledge structure is a form of schema such as those that learners use to represent knowledge in their memory [2]. Identifying learners' knowledge structure is a nontrivial effort to reveal what they understand about a domain of particular expertise. Many researchers prove that a concept map is a graphical tool that can represent individual knowledge structures accurately.

A concept map has been used broadly as a tool for organizing and structuring knowledge in teaching, learning, and assessment. Based on its construction method, the concept map style is classified into two types: (1) open-ended; and (2) closed-ended [3]. The open-ended fashion allows learners to use any concepts and any linking words related to the particular topic in their diagrams. This condition enables the teacher to uncover different knowledge structures across students. In contrast, a closed-ended concept mapping style provides finite concepts, connecting lines, and linking phrases regarding the teacher's map. This approach facilitates automatic assessment by comparing students' and teacher's maps.

Concept maps depict individual ideas that connect two concepts and form a proposition. A proposition is the smallest semantic unit in a concept map that presents particular meaning. Many different ways have been proposed to evaluate the concept map, but the most common categories are quantitatively and qualitatively methods. The quantitative analysis focuses on measuring the number of concept map elements or components, such as concepts, relationships, propositions, and hierarchies. Meanwhile, quality analysis focuses on the scientific quality of concept maps on particular topics.

Novak and Gowin proposed concept maps based on the assimilation theory, which emphasized meaningful learning [4]. Meaningful learning is a process of linking 
new information to relevant concepts contained in a cognitive structure. Achieving meaningful learning requires individuals to have a well-organized knowledge structure to integrate new with existing knowledge in a particular domain context [5]. Meaningful learning can be improved by linking prior concept maps to further related information [6]. The expansion of concept maps is an appropriate way of realizing enhanced meaningful learning and promote students' learning outcomes.

Extended concept mapping is a promoting approach to improve student's achievements in learning. This technique provides related activities that allow the structure of expansion organized and promotes problemsolving. Extending concept mapping activities play an essential task in each phase of the knowledge integrating process through reviewing prior ideas and connections, eliciting missing ideas and relationships, and adding new concepts and relationships [7]. The expansion of existing concept maps is a sound strategy for actualizing enhanced meaningful learning and promote good understanding [8]. Referring to the system design, learning material will be divided into two parts: original and additional parts. Extended concept mapping requires learners to connect the prior original map with a new additional map.

Some previous studies reported the effects of extended concept mapping in learning outcomes. A study in [9] proposed ESB concept mapping as an approach to expand the open-ended technique. The experimental results showed that ESB had a positive effect on learning outcomes consisting of post-test, delayed-test, and a number of propositions [8], [9]. Another type of extensible concept mapping study was also performed by [10], which introduced the EKB map. Although both ESB and EKB have positive effects on students' learning outcomes, there was no information concerning the distribution of quality of students' knowledge structure.

The present study investigates the distribution of knowledge structure using ESB and EKB. This study focused on the spreading of students' achievements in the quality of knowledge structure measured using the quality of propositions. We hypothesize that the EKB promotes students to attain a higher and equitable quality of knowledge structure comparing to the ESB.

\section{LITERATURE REVIEW}

\subsection{Extended Scratch-Build}

The Extended Scratch-Build (ESB) concept map is a graphical tool aimed to support learning process and strengthen meaningful learning [9]. It is a computerbased and client/server concept mapping tool to support and improve learning services. Based on the system architecture that supports it, ESB tools can be implemented in various learning styles, including face- to-face or traditional learning, e-learning, and blended learning. Thus, it can be used to support learning in the COVID-19 pandemic.

ESB inspired by open-ended concept mapping techniques that experienced to capture differences across students' knowledge structures. The open-ended method is a potential approach for reflecting and measuring students' knowledge structure. Still, it has a drawback in the achievement of understanding scores that tends to be less than closed-ended method [11]. ESB attempts to overcome the shortcomings of the usual open-ended method by offering an expansion of a two-phase concept map: Phase 1 and Phase 2.

In Phase 1, students are asked to create a concept map following the first material or original map using an open-ended approach. Students are allowed to add concepts, links, and define propositions according to their understanding of the particular material. Phase 1 represents activities usual open-ended technique.

Furthermore, in Phase 2, students would be requested to expand their original concept map by adding new concepts, relationships, and propositions. Unlike activity in Phase 1, which started making concept maps from scratch or zero, Phase 2 has provided an original map to be expanded in correspond to additional material. In this situation, students were also allowed to modify their previous concept maps if necessary.

Observation results showed that ESB has a positive effect on learning outcomes. Students' understanding increased significantly after employing ESB map, which measured using pre-test and post-test scores. The results of the expansion of additional maps also improved significantly compared to the original map, both quantitatively and qualitatively.

\subsection{Extended Kit-Build}

The Extended Kit-Build (EKB) is a new extension of the Kit-Build (KB) concept map framework. It combines $\mathrm{KB}$ style and usual open-ended style concept mapping. $\mathrm{KB}$ map is a recomposition concept map, which is a subcategory of a closed-ended concept map [12]. The recomposition describes an activity where learners are requested to understand the teacher's understanding. KB style requests a learner to reconstruct a concept map by using provided a set of nodes and links that are prepared by decomposing a teacher's map. Through several practical uses, it has been confirmed that the KB map is effective in promoting learner's comprehension of a learning material that is represented as a teacher's map [3].

EKB increased the ability of $\mathrm{KB}$ through map expansion using the open-ended technique. This approach maintains the advantages of closed-ended in achieving maximum test scores and could uncover 
differences among students' knowledge structure, which usually solely made by open-ended. Thus, there are also two phases of mapping activities in EKB: Phase 1 and Phase 2.

Before starting the EKB activity, the teacher creates a teacher's map. He or she could provide the teacher's map manually or using supportive tools [13]. Next, in Phase 1, learners are asked to reconstruct the teacher's map that has been decomposed by the KB system. The main task of learners is to build a knowledge structure in accordance with teacher perception. This activity is realized by linking relations to concepts to construct concept maps. Learners are not allowed to add other components during concept mapping activities.

Phase 2 EKB is similar to ESB, which is to expand the original map by adding new components. In this situation, learners could add new ideas and relationships related to additional material. The main difference between EKB and ESB lies in the mapping activity in Phase 1, where the EKB uses closed-ended KB while ESB utilizes the open-ended technique.

\section{METHODOLOGY}

\subsection{Participants}

This study involved 55 university students $(65.45 \%$ male, $34.55 \%$ female). Participants came from two homogeneous classes (A and B). Although the participants were familiar with using information and communication technology in learning, they were still novice with concept mapping activities. Randomly, class A was assigned as the control group using the ESB method, and class B was the experimental group with the EKB approach.

\subsection{Context Material}

The present study was conducted in the Database 1 course. The Relational Database topic was chosen and delivered in Indonesian. The material topic consists of four following subtopics: introduction to a relational database (consisting of 192 words), relational keys (consisting of 139 words), formal language (composed of 101 words), and commercial language (consisting of 81 words).

In the EKB framework context, the lecturer defined ten propositions on the teacher's map. Eight propositions related to subtopics 1 and 2 propositions related to subtopic 2. An experienced lecturer who had been teaching for 11 years involved in the experiment.

\subsection{Instrument}

The quality of students' knowledge structure was assessed using the quality of propositions scores. The quality of propositions is considered to be one of the most important criteria [14] and states the quality of individual concept maps. Rating the quality of propositions is relational scoring, which has a vital role and complements structural scoring based on map components [15]. Propositions are components of knowledge and represent fundamental elements in concept maps.

The quality of the propositions scoring method was defined by the lecturer to examine the students' knowledge and understanding [8]. Four level scoring were formulated: $0=$ incorrect; $1=$ partially incorrect; 2 $=$ correct with thin scientific understanding; and $3=$ scientifically correct. The quality rating formulation of these propositions was considered entirely appropriate and able to represent the possibility of all propositions' values.

The quality measurements were assessed manually by the lecturer, who also taught in the classroom. The scoring method was carried out on the students' concept map and then classified to related subtopics to find out the distribution. Equitable distribution across all available subtopics is considered as a remarkable achievement.

\subsection{Procedures}

Participants in the control and experimental groups used the same learning environment and equipment. The main difference between the two groups is the concept mapping approach in Phase 1. Before the experiment was conducted, in a previous course meeting, participants in both groups had been given an introduction to concept maps, including usage practices. The experiment activity of this research is shown in Figure. 1.

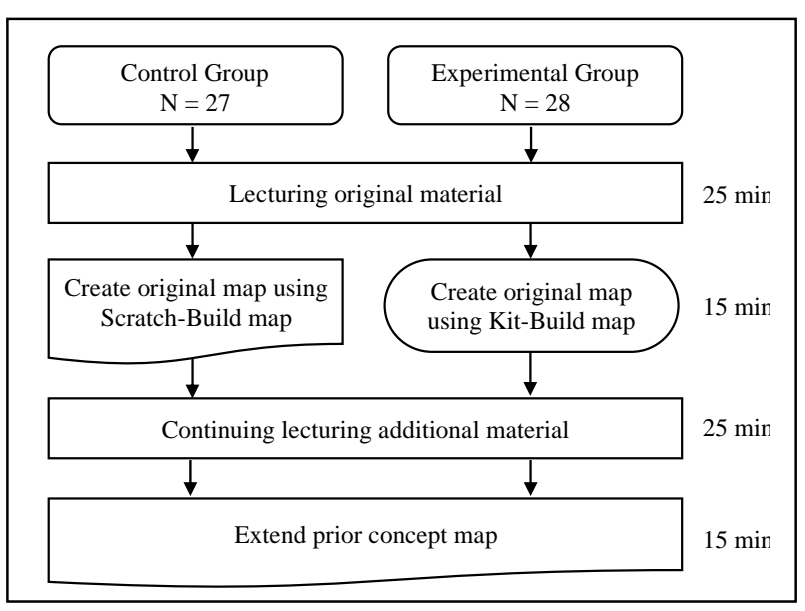

Figure 1 Experiment flow design

The lecturer started the experimental activity by giving original material consisting of 2 subtopics. Learning activity was performed as usual with an allocation of 25 minutes. The lecturer distributed presentation handouts before delivering content. Next is the Phase 1 concept mapping activity for 15 minutes. 
Students in the control group were asked to create a concept map using the scratch-build approach or the open-ended technique, while the experimental group uses the KB map.

In Phase 2, the lecturer continued in giving a lecturing with additional material at the same time, which was 25 minutes. Furthermore, participants in both groups were asked to expand their prior concept maps by adding new components.

\section{RESULTS AND DISCUSSIONS}

\subsection{Analysis of the Distribution of Knowledge Structure}

The present study is focused on the distribution of quality of students' knowledge structure that is measured using the quality of propositions. Referring to the proposition-based scoring method, the lecturer could get the total concept map scores that describe the quality of individuals' knowledge structure. Descriptive statistics of the quality of propositions scores on material subtopics are shown in Table 1 .

There was an exciting pattern based on the results found in the obtained data. In subtopic 1, the average quality of propositions of the control group was slightly higher than the experimental group, 26.33 compared to 23.21. In contrast, in subtopic 2 , the achievement of the experimental group slightly outperformed the control group, which was 16.21 compared to 13.52 .

Furthermore, in subtopic 3, the experimental group seemed to consistently improve its achievement with an average score of 14.68, while the control group was 6.04. The steady performance was seen in subtopic 4 , where students who used EKB were consistent with a high score of 15.79, while students who used ESB obtained 3.22.

TABLE 1. DESCRIPTIVE STATISTICS OF THE QUALITY OF PROPOSITIONS SCORES ON SUBTOPICS MATERIAL

\begin{tabular}{lllllll}
\hline Subtopics & Groups & N & Min & Max & Mean & SD \\
\hline Subtopic 1 & Control & 27 & 10.00 & 52.00 & 26.33 & 9.70 \\
& Experimental & 28 & 20.00 & 25.00 & 23.21 & 1.34 \\
\hline Subtopic 2 & Control & 27 & 1.00 & 29.00 & 13.52 & 7.51 \\
& Experimental & 28 & 12.00 & 30.00 & 16.21 & 4.46 \\
\hline Subtopic 3 & Control & 27 & 2.00 & 18.00 & 6.04 & 3.71 \\
& Experimental & 28 & 6.00 & 24.00 & 14.68 & 4.35 \\
\hline Subtopic 4 & Control & 27 & 0.00 & 9.00 & 3.22 & 3.46 \\
& Experimental & 28 & 0.00 & 35.00 & 15.79 & 8.52 \\
\hline
\end{tabular}

The degree of differences between the quality of propositions scores for both groups was measured using the Mann-Whitney U test. Table 2 depicts the statistical analysis results of the quality of propositions scores on four available subtopics material.

In subtopic 1, the mean rank control group was slightly higher than the experimental group, 30.70 and 25.39 , respectively. Even so, there was no significant difference between the quality of propositions for both groups $(Z=-1.249 ; p=.212>.05)$ with Pearson's $r$ of 0.168 , indicating a small effect size.

In subtopic 2, the results appear to be the opposite. The mean rank of students in the experimental group increased compared to the control group, which was 30.30 compared to 25.61. However, there was also no significant difference between the map quality scores for both groups $(Z=-1.090 ; p=.276>.05)$. These results suggested a small effect size with Pearson's $r$ was -0.168 .

Students in the experimental group that used the EKB map have consistent performance. This condition can be seen in subtopic 3, where they were able to keep the mean rank value stable (39.64), while students in the control group tend to decrease (15.93). Statistical analysis results stated there were significant differences between the quality of propositions for both groups $(Z=-5.518$; $p=$ $.000<.05)$ with Pearson's $r$ of -0.744 , indicating a large effect size.

The suspicion that students in the experimental group have stable performance was increasingly evident. Based on a p-value threshold of 0.05 , there was a statistically significant difference between control and experimental groups $(Z=-5.303 ; p=.000<.05)$ on Subtopic 4 . The results of the analysis reported a large effect size with Pearson's $r-7.715$.

TABLE 2. THE MANN-WHITNEY U TEST RESULTS OF THE QUALITY OF PROPOSITIONS ON MATERIAL SUBTOPICS

\begin{tabular}{llllllll}
\hline Subtopics & Groups & N & $\begin{array}{l}\text { Mean } \\
\text { Rank }\end{array}$ & $\begin{array}{l}\text { Sum of } \\
\text { Ranks }\end{array}$ & & $Z$ & $p$ \\
\hline Subtopic 1 & Control & 27 & 30.70 & 829.00 & 305.000 & -1.249 & .212 \\
& Experimental & 28 & 25.39 & 711.00 & & & \\
\hline Subtopic 2 & Control & 27 & 25.61 & 691.50 & 313.500 & -1.090 & .276 \\
& Experimental & 28 & 30.30 & 848.50 & & & \\
\hline Subtopic 3 & Control & 27 & 15.93 & 430.00 & 52.000 & -5.518 & .000 \\
& Experimental & 28 & 39.64 & 111.00 & & & \\
\hline Subtopic 4 & Control & 27 & 16.41 & 443.00 & 65.000 & -5.303 & .000 \\
& Experimental & 28 & 39.18 & 1097.00 & & & \\
\hline
\end{tabular}

Based on statistical analysis results, it can be seen that the quality of propositions scores in the experimental group was superior to the control group. Students in the experimental group that using EKB were able to achieve optimal scores in each related subtopic material. Students are not only consistent in achieving scores but were also higher than those in control groups that used ESB.

\subsection{The Distribution of Learners' Knowledge Structure}

The distribution of the mean of groups' quality of propositions on material subtopics is shown in Figure. 2. The results on subtopic 1 reported that the control group that used open-ended were able to achieve a higher number of propositions than experimental groups that utilized finite concepts and links. This condition also has 
an impact on the quality of propositions scores that were higher than the experimental group, although it does not show significant differences.

In subtopic 2, students in the experimental group showed positive performance and outperformed the control group scores. The existence of the KB kit on the initial topic encourages learners to produce a vigorous basic concept map structure. Kit plays an essential role as a reference when creating concept maps for related next subtopics. As a result, they were able to achieve optimal scores even though they were not available in terms of subtopics.

The decisive role of the KB kit as a guideline was increasingly seen in achievements in subtopics 3 and 4 . Students in the experimental group could maintain their accomplishments, while those in the control group have decreased. The experimental group who used the EKB approach consistently was able to find and connect new propositions with prior knowledge. In addition, the quality of propositions scores of students in the experimental group was also higher than the control group.

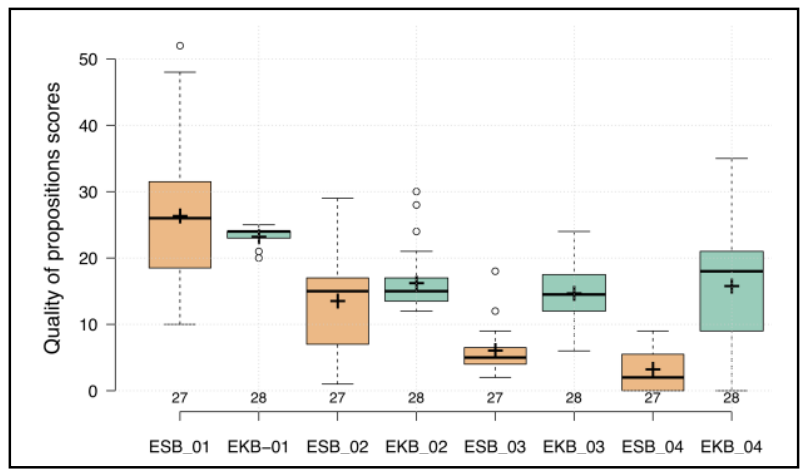

Figure 2 The distribution of the mean of groups' quality of propositions scores regarding material subtopics

The KB approach that uses kits is suitable to support extending activities. A kit represents key concepts defined by the teacher and is a crucial component that plays an essential role in concept mapping [8], [12]. The kit acts as a guideline to help learners in reconstructing the concept map according to the teacher's perception. The existence of a kit accustoms learners to uncover new key concepts and connect with prior knowledge. Kit in Phase 1 triggers students to reveal related ideas in Phase 2 and link them between subtopics. Therefore, experimental groups that use EKB could achieve higher map quality scores and are evenly distributed across all available subtopics materials.

\section{CONCLUSION}

The present study compared the ESB and EKB concept mapping to investigate the distribution of quality of students' knowledge structure. EKB, which combines
KB map and open-ended, has more potential in facilitating and increasing meaningful learning in extending activities.

The EKB approach that used a kit component at the beginning of mapping activities provides an excellent memory and produces a well-organized concept map structure. This finding is proven, in the expansion map phase, students who use EKB have consistent performance and optimal achievement on subtopic material even though it is not related to the teacher's map. Students' in the experimental group not only outperformed ESB in terms of the average of map quality scores attainment but also had a more even distribution of mastery in all available material subtopics.

To acquire more reliable experimental results, further works should involve a higher number of participants and suggest several topics. Information related learners' behavior in creating concept maps will also be useful evidence. Future studies could include log data and analyze it to provide appealing details.

\section{AUTHORS' CONTRIBUTIONS}

DDP designed and developed the system. DDP and AP designed, prepared, and conducted the experiment under the supervision of $\mathrm{YH}$ and TH. DDP and AP analyzed the data and prepared the manuscript.

\section{ACKNOWLEDGMENTS}

The first author would like to acknowledge the Islamic Development Bank (IsDB), the Universitas Negeri Malang (UM), Indonesia, and the Dept. of Information Engineering, Hiroshima University, Japan, for invaluable supports.

\section{REFERENCES}

[1] M. A. Ruiz-Primo, "Examining concept maps as an assessment tool," Proc. of the First Int. Conference on Concept Mapping Pamplona, Spain 2004.

[2] M. D. Merrill, "Knowledge objects and mental models," Proceedings International Workshop on Advanced Learning Technologies. IWALT 2000. IEEE 2000

[3] T. Hirashima, "Reconstructional Concept Map: Automatic Assessment and Reciprocal reconstruction," The International Journal of Innovation, Creativity and Change (IJICC), 5 (5), 669-682, 2019.

[4] J. D. Novak, "Learning, creating, and using knowledge: concept maps as facilitative tools in schools and corporations (2nd ed.)". New York: Routledge. 2010 
[5] J. D. Novak and A. J. Cañas, "The theory underlying concept maps and how to construct them," Technical Report IHMC CmapTools 2006-01 Rev 01-2008, Florida Institute for Human and Machine Cognition, 2008

[6] R. Boffey, P. Gerrans, and S. Kennedy, "Using digital lectures to assist student learning," eCULTURE, 3(1), 17, 2010.

[7] B. A. Schwendimann and M. C. Linn, "Comparing two forms of concept map critique activities to facilitate knowledge integration processes in evolution education," Journal of Research in Science Teaching, 53(1), 70-94, 2016.

[8] D. D. Prasetya, T. Hirashima, and Y. Hayashi, "Study on Extended Scratch-Build Concept Map to Enhance Students' Understanding and Promote Quality of Knowledge Structure," International Journal of Advanced Computer Science and Applications(IJACSA), $11(4), \quad 2020$. http://dx.doi.org/10.14569/IJACSA.2020.0110420

[9] D. D. Prasetya, T. Widiyaningtyas, S. C. Putro, T. Hirashima, and Y. Hayashi, "Extended ScratchBuild Concept Map to Enhance Meaningful Learning," International Conference on Electrical, Electronics and Information Engineering (ICEEIE) (Vol. 6, pp. 187-191), IEEE, 2019. https://doi.org/10.1109/ICEEIE47180.2019.898143 5

[10] D. D. Prasetya, T. Hirashima, and Y. Hayashi, "KBMixed: A Reconstruction and Improvable Concept Map to Enhance Meaningful Learning and Knowledge Structure," Proceedings of The 26th International Conference on Computers in Education (ICCE 2019) December, 809-812, 2019.

[11] M. A. Ruiz - Primo, S. E. Schultz, M. Li, and R. J. Shavelson, "Comparison of the reliability and validity of scores from two concept - mapping techniques", Journal of Research in Science Teaching: The Official Journal of the National Association for Research in Science Teaching, 38(2), 260-278, 2001

[12] T. Hirashima, K. Yamasaki, H. Fukuda, and H. Funaoi, "Framework of kit-build concept map for automatic diagnosis and its preliminary use," Research and Practice in Technology Enhanced Learning, 10 (1), 17, 2015.

[13] A. Pinandito, H.M. Az-zahra, T. Hirashima, Y. Hayashi, "User experience evaluation on computersupported concept map authoring tool of kit-build concept map framework. 2019 International Conference on Sustainable Information Engineering and Technology (SIET 2019), 2019, pp. 289-294. https://doi.org/10.1109/SIET48054.2019.8986005

[14] Z. Raud, V. Vodovozov, and T. Lehtla, "Teaching, Learning, and Assessment Integration in Electronics on the Concept Map Basis," in Innovating with Concept Mapping Proceedings of the Seventh International Conference on Concept Mapping, Tallinn, Estonia 2016, pp. 199-207

[15] J. M. Walker and P. H. King, "Concept mapping as a form of student assessment and instruction in the domain of bioengineering," Journal of Engineering Education, 92(2), 167-178. 2003. 